$\underline{\text { Preprint typeset in JHEP style - HYPER VERSION }}$

hep-th/0412174

KAIST-TH 2004/21

\title{
Gauged $U(1)_{R}$ Symmetries and Fayet-Iliopoulos Terms in 5D Orbifold Supergravity
}

\author{
Hiroyuki Abe and Kiwoon Choi \\ Department of Physics, Korea Advanced Institute of Science and Technology, Daejeon \\ 305-701, Korea \\ E-mail: abe@hep.kaist.ac.kr, kchoi@hep.kaist.ac.kr
}

\begin{abstract}
We discuss a gauged $U(1)_{R}$ supergravity on five-dimensional (5D) orbifold $\left(S^{1} / Z_{2}\right)$ in which both a $Z_{2}$-even $U(1)$ gauge field and the $Z_{2}$-odd graviphoton take part in the $U(1)_{R}$ gauging. Based on the off-shell formulation of $5 \mathrm{D}$ supergravity, we analyze the structure of Fayet-Iliopoulos (FI) terms allowed in such model. Introducing a $Z_{2}$-even $U(1)_{R}$ gauge field accompanies new bulk and boundary FI terms in addition to the known integrable boundary FI term which could be present in the absence of any gauged $U(1)_{R}$ symmetry. Some physical consequences of these new FI terms are examined.
\end{abstract}

KEywords: Field Theories in Higher Dimensions, Supergravity Models. 


\section{Contents}

1. Introduction 1

2. 5D orbifold supergravity with gauged $U(1)_{R}$

3. 4D Poincaré invariant solutions 9

3.1 Killing conditions and 4D energy functional 9

3.2 Vacuum solutions with $U(1)_{R}$ FI terms 11

4. Conclusion 16

\section{Introduction}

Supersymmetry (SUSY) is an attractive candidate for physics beyond the standard model (SM) as it can stabilize the electroweak scale against the high energy radiative corrections to the Higgs boson mass. Furthermore SUSY is a fundamental ingredient of the only known consistent theory of quantum gravity, i.e., superstring theory. To be phenomenologically viable, SUSY should be broken by a nonzero vacuum value of $F$ and/or $D$ auxiliary component. This can be achieved by introducing a tadpole term for the $F$ and/or $D$-component in the effective Lagrangian, for instance an O'Raifeartaigh term for F-breaking [1] and a Fayet-Iliopoulos (FI) term for $D$-breaking [2]. However in locally supersymmetric theory $D$-term SUSY breaking is severely limited since FI term is not allowed unless the corresponding $U(1)$ is either an $R$-symmetry [3] or a so-called pseudo-anomalous $U(1)$ symmetry with Green-Schwarz anomaly cancellation mechanism [何.

Recently five-dimensional (5D) supergravity (SUGRA) on the orbifold $S^{1} / Z_{2}$ has been studied as an interesting theoretical framework for physics beyond the SM. It has been noted that $5 \mathrm{D}$ orbifold SUGRA with a $U(1)_{R}$ symmetry gauged by the $Z_{2}$-odd graviphoton can provide the supersymmetric Randall-Sundrum (RS) model [5] in which the weak to Planck scale hierarchy can arise naturally from the geometric localization of $4 \mathrm{D}$ graviton [6]. In this case, the bulk cosmological constant and brane tensions which are required to generate the necessary $\mathrm{AdS}_{5}$ geometry appear in the Lagrangian as a consequence of the $U(1)_{R}$ FI term with $Z_{2}$-odd coefficient.

In this paper we consider a more generic orbifold SUGRA which contains a $Z_{2}$-even $5 \mathrm{D}$ gauge field $A_{\mu}^{X}$ participating in the $U(1)_{R}$ gauging. If $4 \mathrm{D} N=1 \mathrm{SUSY}$ is preserved by the compactification, the $4 \mathrm{D}$ effective theory of such model will contain a gauged $U(1)_{R}$ symmetry associated with the zero mode of $A_{\mu}^{X}$, which is not the case when the $5 \mathrm{D} U(1)_{R}$ is gauged only through the $Z_{2}$-odd graviphoton. Based on the known off-shell formulation [7], we formulate a gauged $U(1)_{R}$ SUGRA on $S^{1} / Z_{2}$ in which both $A_{\mu}^{X}$ and the graviphoton 
take part in the $U(1)_{R}$ gauging and then analyze the structure of FI terms allowed in such model. As expected, introducing a $Z_{2}$-even $U(1)_{R}$ gauge field accompanies new bulk and boundary FI terms in addition to the known integrable boundary FI term which could be present in the absence of any gauged $U(1)_{R}$ symmetry [8, 9, 10, 11]. As we will see, those new FI terms can have interesting implications to the quasi-localization of the matter zero modes in extra dimension [12] and also to the SUSY breaking and radion stabilization.

The organization of this paper is as follows. In the next section we provide a formulation for $U(1)_{R}$-gauged orbifold SUGRA based on the Kugo-Ohashi off-shell SUGRA formulation [7]. In section 3, we discuss the conditions for unbroken $N=1$ SUSY for generic 4D Poincaré invariant field configurations and also some physical consequences of the FI terms of the $Z_{2}$-even $U(1)_{R}$ gauge symmetry. Section $\mathbb{\theta}$ is a conclusion.

\section{5D orbifold supergravity with gauged $U(1)_{R}$}

In this section we provide a formulation for 5D SUGRA on $S^{1} / Z_{2}$ in which both a $Z_{2}$-even gauge field and the $Z_{2}$-odd graviphoton take part in the $U(1)_{R}$ gauging. As a minimal example, we consider a model containing three $U(1)$ vector multiplets:

$$
\begin{aligned}
& \mathcal{V}_{Z}=\left(\alpha, A_{\mu}^{Z}, \Omega^{Z i}, Y^{Z i j}\right), \\
& \mathcal{V}_{X}=\left(\beta, A_{\mu}^{X}, \Omega^{X i}, Y^{X i j}\right), \\
& \mathcal{V}_{S}=\left(\gamma, A_{\mu}^{S}, \Omega^{S i}, Y^{S i j}\right),
\end{aligned}
$$

together with a compensator hypermultiplet $\mathcal{H}_{c}$ and physical hypermultiplets $\mathcal{H}_{p}$ :

$$
\begin{aligned}
& \mathcal{H}_{c}=\left(\mathcal{A}_{i}^{x}, \eta^{x}, \mathcal{F}_{i}^{x}\right), \\
& \mathcal{H}_{p}=\left(\Phi_{i}^{x}, \zeta^{x}, F_{i}^{x}\right),
\end{aligned}
$$

where $M^{A}=(\alpha, \beta, \gamma)(A=Z, X, S)$ are real scalar components, $\Omega_{A}^{i}(i=1,2)$ are $S U(2) \mathbf{U}^{-}$ doublet symplectic Majorana spinors, $Y^{A i j}=Y^{A j i}$ are $S U(2)$ U-triplet auxiliary components, $\mathcal{A}_{i}^{x}, \Phi_{i}^{x}(x=1,2)$ are quaternionic hyperscalars, $\eta^{x}, \zeta^{x}$ are symplectic Majorana hyperinos, and $\mathcal{F}_{i}^{x}, F_{i}^{x}$ are the auxiliary components of hypermultiplets. Here $\mathcal{V}_{Z}$ is the model-independent central charge vector multiplet which contains the $Z_{2}$-odd graviphoton $A_{\mu}^{Z}, \mathcal{V}_{X}$ is a vector multiplet which contains a $Z_{2}$-even $U(1)$ gauge field $A_{\mu}^{X}$, and $\mathcal{V}_{S}$ is a non-physical vector multiplet which is introduced to obtain the necessary $Z_{2}$-odd couplings through the 4 -form multiplier mechanism of Ref. [13]. The $Z_{2}$-parities of these components are summarized in Table 1 .

Because we have a single compensator hypermultiplet $\mathcal{H}_{c}$, the corresponding quaternionic manifold spanned by physical hyperscalars $\mathcal{H}_{p}\left(p=1,2, . ., n_{H}\right)$ is $U S p\left(2,2 n_{H}\right) / U S p(2) \times$ $U S p\left(2 n_{H}\right)$. In the following, we will use frequently a matrix notation for hyperscalars, e.g.,

$$
\Phi \equiv\left(\begin{array}{cc}
\Phi_{i=1}^{x=1} & \Phi_{i=2}^{x=1} \\
\Phi_{i=1}^{x=2} & \Phi_{i=2}^{x=2}
\end{array}\right)=\left(\begin{array}{cc}
\Phi_{+} & \Phi_{-} \\
-\Phi_{-}^{*} & \Phi_{+}^{*}
\end{array}\right),
$$

where $\Phi_{ \pm}$are $Z_{2}$ parity eigenstates, and the similar notation is adopted for the compensator $\mathcal{A}$. In this matrix notation, the symplectic reality condition and the $Z_{2}$ boundary condition 


\begin{tabular}{c|c|c}
$Z_{2}$-even & $\alpha, A_{\mu}^{X, S}, A_{y}^{Z},\left(Y^{X, S}\right)^{(3)},\left(Y^{Z}\right)^{(1,2)}$ & $(\mathcal{A}, \Phi)_{i=1}^{x=1},(\mathcal{A}, \Phi)_{i=2}^{x=2},(\mathcal{F}, F)_{i=2}^{x=1},(\mathcal{F}, F)_{i=1}^{x=2}$ \\
\hline$Z_{2}$-odd & $\beta, \gamma, A_{\underline{\mu}}^{Z}, A_{y}^{X, S},\left(Y^{Z}\right)^{(3)},\left(Y^{X, S}\right)^{(1,2)}$ & $(\mathcal{A}, \Phi)_{i=2}^{x=1},(\mathcal{A}, \Phi)_{i=1}^{x=2},(\mathcal{F}, F)_{i=1}^{x=1},(\mathcal{F}, F)_{i=2}^{x=2}$
\end{tabular}

Table 1: The $Z_{2}$-parity assignment of component fields. Here $\underline{\mu}=0,1,2,3$, while $y$ is the fifth coordinate of $S^{1} / Z_{2}$ and $Y_{j}^{A i}=\sum_{r=1}^{3}\left(i \sigma_{r}\right)^{i}{ }_{j}\left(Y^{A}\right)^{(r)}(A=Z, X, \bar{S})$.

are given by

$$
\Phi^{*}(y)=i \sigma_{2} \Phi(y) i \sigma_{2}^{T}, \quad \Phi(-y)=\sigma_{3} \Phi(y) \sigma_{3}
$$

We will also use a matrix notation for the $S U(2)_{\mathbf{U}}$-triplet fields as, e.g., $Y^{A} \equiv Y_{j}^{A i}$. Note that the $S U(2)_{\mathbf{U}}$ index is raised and lowered by an antisymmetric tensor $\epsilon_{i j}=\epsilon^{i j}=i \sigma_{2}$ like, e.g., $Y_{j}^{A i}=Y^{A i k} \epsilon_{k j}=\epsilon^{i k} Y_{k j}^{A}$.

The most general hypermultiplet gauging consistent with the $Z_{2}$ orbifolding and the symplectic reality condition (1) is given by

$$
\begin{aligned}
& \left(T_{Z}, T_{X}, T_{S}\right) \Phi=(0, q, c) i \sigma_{3} \Phi \\
& \left(T_{Z}, T_{X}, T_{S}\right) \mathcal{A}=\left(0,-r,-\frac{3}{2} k\right) i \sigma_{3} \mathcal{A}
\end{aligned}
$$

where $T_{Z, X, S}$ are the $U(1)_{Z, X, S}$ generators and $q, c, r, k$ are real constants. The vector multiplet part of 5D SUGRA is determined by the norm function $\mathcal{N}$ which is a homogeneous cubic polynomial of $M^{A}: \mathcal{N}=C_{A B C} M^{A} M^{B} M^{C}$ for a totally symmetric constants $C_{A B C}$. In this paper, we choose

$$
\mathcal{N}=\alpha^{3}-\frac{1}{2} \alpha \beta^{2}+\frac{1}{2} \xi_{F I} \alpha \beta \gamma,
$$

which corresponds to a minimal model for our purpose.

Under the constraint on $\mathcal{V}_{S}$ induced by the four-form multiplier field which will be introduced below, a nonzero $\xi_{F I}$ gives an integrable boundary FI term for the $U(1)_{X}$ vector multiplet: $-\frac{1}{2} \xi_{F I} Y^{X(3)} \partial_{y} \epsilon(y)$ where we use an isovector notation $Y^{A}=\sum_{r=1}^{3}\left(i \sigma_{r}\right) Y^{A(r)}$, the gauge charge $k$ of the compensator gives a negative bulk cosmological constant $-6 k^{2}$ for $\mathrm{AdS}_{5}$ geometry as well as the correct integrable boundary tension $3 k \partial_{y} \epsilon(y)$, and finally the gauge charge $c$ of the physical hypermultiplet gives a hyperino kink mass $c \epsilon(y)$. The four-form multiplier mechanism of Ref. [13] provides a dynamical way to generate $Z_{2}$-odd couplings proportional to the periodic sign-function $\epsilon(y)=y /|y|$ which obeys

$$
\begin{aligned}
& \epsilon(y)=-\epsilon(-y)=\epsilon(y+2 \pi R)=1 \quad(0<y<\pi R), \\
& \partial_{y} \epsilon(y)=2[\delta(y)-\delta(y-\pi R)] .
\end{aligned}
$$

To implement the four-form mechanism within off-shell SUGRA, we introduce a multiplier multiplet defined under a background of $\mathcal{V}_{Z}$ :

$$
L_{H}=\left(L^{i j}, \varphi^{i}, E_{\mu \nu \rho}, H_{\mu \nu \rho \sigma}\right) \text {, }
$$


where $L^{i j}$ is a $S U(2)$-triplet scalar, $\varphi^{i}$ is a $S U(2)$-doublet fermion, $E_{\mu \nu \rho}$ and $H_{\mu \nu \rho \sigma}$ are three and four-form fields, respectively. The off-shell action of this multiplier multiplet was derived in Ref. [7] as

$$
\begin{aligned}
& \mathcal{L}_{4-\text { form }}^{\text {bulk }}=e\left(Y^{S i j}-G Y^{Z i j}\right) L_{i j}-\frac{1}{4 !} \epsilon^{\lambda \mu \nu \rho \sigma}\left[\left\{F_{\lambda \mu}\left(A^{S}\right)-G F_{\lambda \mu}\left(A^{Z}\right)\right\} E_{\nu \rho \sigma}+\frac{1}{2} G \partial_{\lambda} H_{\mu \nu \rho \sigma}\right], \\
& \mathcal{L}_{4-\text { form }}^{\text {brane }}=\left(a_{0} \delta(y)+a_{\pi} \delta(y-\pi R)\right)\left[\frac{1}{4 !} \epsilon^{\mu \nu \rho \sigma y} H_{\mu \nu \rho \sigma}+e_{(4)} \alpha \epsilon^{j k}\left(i \sigma_{3}\right)^{i}{ }_{k} L_{i j}\right]
\end{aligned}
$$

where $\epsilon^{j k}$ is the $S U(2)$-invariant antisymmetric tensor, $G=M^{S} / M^{Z}=\gamma / \alpha, e=\left(-\operatorname{det}\left(g_{\mu \nu}\right)\right)^{1 / 2}$, and $e_{(4)}=\left(-\operatorname{det}\left(g_{\underline{\mu} \underline{\nu}}\right)\right)^{1 / 2}$ for the induced $4 \mathrm{D}$ metric $g_{\underline{\mu} \underline{\underline{\nu}}}$ on the boundaries. Then the equations of motion for $H_{\mu \nu \rho \sigma}, E_{\mu \nu \rho}$ and $L_{i j}$ give

$$
\begin{aligned}
G & =\gamma / \alpha=\epsilon(y), \\
F_{\mu \nu}\left(A^{S}\right) & =\epsilon(y) F_{\mu \nu}\left(A^{Z}\right), \\
Y^{S i j} & =\epsilon(y) Y^{Z i j}+e^{-1} e_{(4)} \alpha \epsilon^{j k}\left(i \sigma_{3}\right)_{k}^{i}(\delta(y)-\delta(y-\pi R)),
\end{aligned}
$$

where the integrability condition of $\partial_{y} G$ results in $a_{0}=-a_{\pi}=-1 / 2$. Now using the relations in (2.4), the redundant vector multiplet $\mathcal{V}_{S}$ can be replaced by the central charge vector multiplet $\mathcal{V}_{Z}$ multiplied by the $Z_{2}$-odd factor $\epsilon(y)$. This four-form mechanism provides an elegant way to obtain a locally supersymmetric theory of $\mathcal{V}_{I}(I=Z, X)$ involving $Z_{2}$-odd couplings, e.g., $\xi_{F I} \epsilon(y), k \epsilon(y)$ and $c \epsilon(y)$ in our case, starting from a locally supersymmetric theory of $\mathcal{V}_{A}(A=Z, X, S)$ and the four-form multiplier multiplet involving only $Z_{2}$-even couplings.

Upon ignoring the UV-sensitive higher-dimensional boundary terms, after integrating out the Lagrange multipliers and also the auxiliary fields other than $Y^{I i j}, V_{\mu}^{i j}, v_{\mu \nu}$ and $\mathcal{F}_{i}^{x}$ (here $V_{\mu}^{i j}$ and $v_{\mu \nu}=-v_{\nu \mu}$ are the auxiliary fields in the Weyl multiplet of 5D conformal SUGRA), we find the following Lagrangian density of bosonic fields:

$$
\begin{aligned}
\mathcal{L}= & \mathcal{L}_{\text {bulk }}+\mathcal{L}_{\text {brane }}+\mathcal{L}_{\text {aux }} \\
e^{-1} \mathcal{L}_{\text {bulk }}= & -\frac{1}{2} R-\frac{1}{4} \tilde{a}_{I J} F_{\mu \nu}^{I} F^{\mu \nu J}+\frac{1}{2} \tilde{a}_{I J} \nabla_{\mu} M^{I} \nabla^{\mu} M^{J} \\
& +\frac{1}{8} e^{-1} \tilde{C}_{I J K} \epsilon^{\lambda \mu \nu \rho \sigma} A_{\lambda}^{I} F_{\mu \nu}^{J} F_{\rho \sigma}^{K}+\operatorname{tr}\left[\nabla_{\mu} \Phi \nabla^{\mu} \Phi^{\dagger}-\nabla_{\mu} \mathcal{A} \nabla^{\mu} \mathcal{A}^{\dagger}\right. \\
& \left.-V_{\mu \text { sol }}^{\dagger} V_{\text {sol }}^{\mu}-M^{I} M^{J}\left(\Phi^{\dagger} t_{I}^{\dagger} t_{J} \Phi-\mathcal{A}^{\dagger} t_{I}^{\dagger} t_{J} \mathcal{A}\right)\right] \\
& -\frac{1}{2} \operatorname{tr}\left[\tilde{\mathcal{N}}_{I J} Y^{I \dagger} Y^{J}-4 Y^{I \dagger}\left(\mathcal{A}^{\dagger} t_{I} \mathcal{A}-\Phi^{\dagger} t_{I} \Phi\right)\right] \\
e_{(4)}^{-1} \mathcal{L}_{\text {brane }}= & {\left[\frac{1}{2} \xi_{F I} \alpha^{2}\left(\operatorname{tr}\left[i \sigma_{3} Y^{X}\right]+e^{-1} e_{(4)} \partial_{y} \beta\right)+\frac{1}{2} \xi_{F I} \alpha \beta \operatorname{tr}\left[i \sigma_{3} Y^{Z}\right]\right.} \\
& \left.-2 \alpha\left(3 k+\frac{3}{2} k \operatorname{tr}\left[\Phi^{\dagger} \Phi\right]+c \operatorname{tr}\left[\Phi^{\dagger} \sigma_{3} \Phi \sigma_{3}\right]\right)\right](\delta(y)-\delta(y-\pi R)) \\
e^{-1} \mathcal{L}_{\text {aux }}= & -\left(V_{\mu}-V_{\mu \text { sol }}\right)^{i j}\left(V^{\mu}-V_{\text {sol }}^{\mu}\right)_{i j}+\left(1-\alpha^{-2} A_{\mu}^{Z} A^{Z \mu}\right) \operatorname{tr}\left[\left(\mathcal{F}-\mathcal{F}_{\text {sol }}\right)^{\dagger}\left(\mathcal{F}-\mathcal{F}_{\text {sol }}\right)\right] \\
& +2\left(v-v_{\text {sol }}\right)^{\mu \nu}\left(v-v_{\text {sol }}\right)_{\mu \nu},
\end{aligned}
$$


where

$$
\begin{aligned}
\tilde{\mathcal{N}} & =\left.\mathcal{N}\right|_{\gamma=\epsilon(y) \alpha}=\tilde{C}_{I J K} M^{I} M^{J} M^{K}=\alpha^{3}-\frac{1}{2} \alpha \beta^{2}+\frac{1}{2} \xi_{F I} \epsilon(y) \alpha^{2} \beta \\
\tilde{a}_{I J} & =-\frac{1}{2} \frac{\partial^{2} \ln \tilde{\mathcal{N}}}{\partial M^{I} \partial M^{J}}=\left(\begin{array}{c}
\tilde{a}_{Z Z} \tilde{a}_{Z X} \\
\tilde{a}_{X Z} \tilde{a}_{X X}
\end{array}\right) \\
\tilde{a}_{Z Z} & =\frac{1}{8 \tilde{\mathcal{N}}^{2}}\left(\beta^{4}+2 \alpha\left(6 \alpha^{3}+4 \xi_{F I} \epsilon(y) \alpha^{2} \beta-\xi_{F I} \epsilon(y) \beta^{3}+\xi_{F I}^{2} \epsilon^{2}(y) \alpha \beta^{2}\right)\right), \\
\tilde{a}_{X Z} & =\tilde{a}_{Z X}=\frac{1}{8 \tilde{\mathcal{N}}^{2}} \alpha^{2}\left(-8 \alpha \beta+\xi_{F I} \epsilon(y)\left(2 \alpha^{2}-\beta^{2}\right)\right), \\
\tilde{a}_{X X} & =\frac{1}{8 \tilde{\mathcal{N}}^{2}} \alpha^{2}\left(2 \beta^{2}-2 \xi_{F I} \epsilon(y) \alpha \beta+\left(4+\xi_{F I}^{2} \epsilon^{2}(y)\right) \alpha^{2}\right), \\
V_{\mu \mathrm{sol}} & =-\frac{1}{2}\left(\mathcal{A}^{\dagger}\left(\nabla_{\mu} \mathcal{A}\right)-\left(\nabla_{\mu} \mathcal{A}\right)^{\dagger} \mathcal{A}\right)+\frac{1}{2}\left(\Phi^{\dagger}\left(\nabla_{\mu} \Phi\right)-\left(\nabla_{\mu} \Phi\right)^{\dagger} \Phi\right), \\
\mathcal{F}_{\text {sol }} & =\alpha t_{Z} \mathcal{A}, \quad v_{\mu \nu \text { sol }}=-\frac{1}{4 \tilde{\mathcal{N}}^{2}} \tilde{\mathcal{N}}_{I} F_{\mu \nu}\left(A^{I}\right) .
\end{aligned}
$$

Here $\tilde{\mathcal{N}}_{I}=\frac{\partial \tilde{\mathcal{N}}}{\partial M^{T}}, \tilde{\mathcal{N}}_{I J}=\frac{\partial^{2} \tilde{\mathcal{N}}}{\partial M^{I} \partial M^{J}}$ for $M^{I}=(\alpha, \beta)(I=Z, X)$ and

$$
\tilde{\mathcal{N}}^{I J}=\frac{1}{\beta^{2}-\xi_{F I} \epsilon(y) \alpha \beta+\left(6+\xi_{F I}^{2} \epsilon^{2}(y)\right) \alpha^{2}}\left(\begin{array}{cc}
\alpha & -\beta+\xi_{F I} \epsilon(y) \alpha \\
-\beta+\xi_{F I} \epsilon(y) \alpha & -6 \alpha-\xi_{F I} \epsilon(y) \beta
\end{array}\right),
$$

is the inverse matrix of $\tilde{\mathcal{N}}_{I J}$. Note that after the multiplier multiplet (2.2) is integrated out, the new $U(1)$ generators $t_{I}$ for hyperscalars are given by

$$
\begin{aligned}
& \left(t_{Z}, t_{X}\right) \Phi=(c \epsilon(y), q) i \sigma_{3} \Phi, \\
& \left(t_{Z}, t_{X}\right) \mathcal{A}=\left(-\frac{3}{2} k \epsilon(y),-r\right) i \sigma_{3} \mathcal{A},
\end{aligned}
$$

Here we have already integrated out the auxiliary fields other than $Y^{I i j}, V_{\mu}^{i j}, v_{\mu \nu}$ and $\mathcal{F}_{i}^{x}$ which we keep at off-shell values since the on-shell values of these auxiliary fields are affected by the boundary supergravity which will be discussed at the end of this section. The complete form of $\mathcal{L}_{\text {aux }}$ involving all auxiliary components can be found in Ref. [7].

The $2 \times 2$ matrix valued compensator hyperscalar field can be chosen as

$$
\mathcal{A} \equiv \mathbf{1}_{2} \sqrt{1+\frac{1}{2} \operatorname{tr}\left[\Phi^{\dagger} \Phi\right]}
$$

in the unit with the $5 \mathrm{D}$ Planck mass $M_{5}=1$, which corresponds to the $S U(2)_{\mathbf{U}}$ gauge fixing condition in the hypermultiplet compensator formulation of off-shell 5D SUGRA [7]. Also by the dilatation gauge fixing condition the norm function can be fixed as

$$
\tilde{\mathcal{N}} \equiv 1
$$

Then we have only one physical gauge scalar field $\phi$ in our system which parameterizes the original scalar fields $\alpha$ and $\beta$ in $\tilde{\mathcal{N}}$ as

$$
\alpha=\frac{\cosh ^{2 / 3}(\phi)}{\left(1+\xi_{F I}^{2} \epsilon^{2}(y) / 8\right)^{1 / 3}}
$$




$$
\begin{aligned}
& =1+\frac{1}{3}\left(\phi^{2}-\frac{1}{8} \xi_{F I}^{2} \epsilon^{2}(y)\right)+\mathcal{O}\left(\phi^{4}\right), \\
\beta & =\frac{\cosh ^{2 / 3}(\phi)\left[\left(2+\xi_{F I}^{2} \epsilon^{2}(y) / 4\right)^{1 / 2} \tanh (\phi)+\xi_{F I} \epsilon(y) / 2\right]}{\left(1+\xi_{F I}^{2} \epsilon^{2}(y) / 8\right)^{1 / 3}} \\
& =\frac{1}{2} \xi_{F I} \epsilon(y)+\sqrt{2} \phi+\mathcal{O}\left(\phi^{3}\right) .
\end{aligned}
$$

The very special manifold spanned by $\phi$ has the metric

$$
\begin{aligned}
g_{\phi \phi}(\phi) & =-\frac{1}{2} \frac{\partial^{2} \ln \tilde{\mathcal{N}}}{\partial M^{I} \partial M^{J}} \frac{\partial M^{I}}{\partial \phi} \frac{\partial M^{J}}{\partial \phi} \\
& =\frac{1+2 \cosh (2 \phi)}{3 \cosh ^{2}(\phi)}=1+\frac{1}{3} \phi^{2}+\mathcal{O}\left(\phi^{4}\right) .
\end{aligned}
$$

Obviously, $\phi$ is $Z_{2}$-odd for $Z_{2}$-odd $\beta$.

Let us identify the gauged $U(1)_{R}$ symmetries of the model. We note that $S U(2)_{R}$ representation is labelled by the $i, j$ indices of component fields after the $S U(2) \mathbf{U}$ gauge fixing (2.10). In this setting, gauging the $R$-symmetry corresponds to making the compensator hypermultiplet to have a nonzero gauge coupling. If the compensator couples to a physical gauge field $A_{\mu}^{R}$, the covariant derivative is given by

$$
\mathcal{D}_{\mu} \mathcal{A}_{i}^{x}=\partial_{\mu} \mathcal{A}_{i}^{x}-\left(V_{\mu}\right)_{i j} \mathcal{A}^{x j}-\left(A_{\mu}^{R}\right)_{y}^{x} \mathcal{A}_{i}^{y}
$$

where $\left(A_{\mu}^{R}\right)_{y}^{x}=-\left(r A_{\mu}^{X}+\frac{3}{2} k \epsilon(y) A_{\mu}^{Z}\right)\left(i \sigma_{3}\right)_{y}^{x}$ in our case, and $V_{\mu}$ is the auxiliary $S U(2)_{\mathbf{U}}$ gauge field in the SUGRA (Weyl) multiplet. After the compensator gauge fixing (2.10), the auxiliary $S U(2) \mathbf{U}$ gauge field is redefined as 14

$$
\left(V_{\mu}^{N}\right)^{i}{ }_{j}=\left(V_{\mu}\right)^{i}{ }_{j}-\left(A_{\mu}^{R}\right)^{i}{ }_{j}=\left(V_{\mu}\right)^{i}{ }_{j}+\left(r A_{\mu}^{X}+\frac{3}{2} k \epsilon(y) A_{\mu}^{Z}\right)\left(i \sigma_{3}\right)^{i}{ }_{j},
$$

yielding the $R$-gauge couplings of all $S U(2)_{\mathbf{U}}$ non-singlet physical fields, e.g.,

$$
\begin{aligned}
\mathcal{D}_{\nu} \psi_{\mu}^{i} & =\nabla_{\nu} \psi_{\mu}^{i}-\left(V_{\nu}\right)^{i}{ }_{j} \psi_{\mu}^{j} \\
& =\nabla_{\nu} \psi_{\mu}^{i}-\left(V_{\nu}^{N}\right)^{i}{ }_{j} \psi_{\mu}^{j}+\left(r A_{\nu}^{X}+\frac{3}{2} k \epsilon(y) A_{\nu}^{Z}\right)\left(i \sigma_{3}\right)^{i}{ }_{j} \psi_{\mu}^{j},
\end{aligned}
$$

for the gravitino $\psi_{\mu}^{i}$. Therefore when $r \neq 0$, the $Z_{2}$-even $A_{\mu}^{X}$ becomes a $U(1)_{R}$ gauge field in the $\sigma_{3}$ direction of $S U(2)_{R}$, while for $k \neq 0$ the $Z_{2}$-odd graviphoton $A_{\mu}^{Z}$ becomes a $U(1)_{R}$ gauge field again in the $\sigma_{3}$ direction. In this prescription, the $Z_{2}$-even (odd) hyperscalar $\Phi_{+}\left(\Phi_{-}\right)$carries a $U(1)_{X}$ charge $q+r(q-r)$, while its fermionic partner carries a $U(1)_{X}$ charge $q$.

The model described by (2.5), (2.6) and (2.7) contain various FI terms which are linear in the auxiliary components $Y^{Z, X}$. For instance, there appear the boundary FI terms of $Y^{X, Z}$ which arise from the $\xi_{F I^{-}}$term in $\tilde{\mathcal{N}}[9]$ :

$$
\frac{1}{2} \xi_{F I}\left[\alpha^{2}\left(\operatorname{tr}\left[i \sigma_{3} Y^{X}\right]+e_{4}^{y} \partial_{y} \beta\right)+\alpha \beta \operatorname{tr}\left[i \sigma_{3} Y^{Z}\right]\right](\delta(y)-\delta(y-\pi R)),
$$


The first $U(1)_{X}$ FI term has been discussed extensively in the literatures [8, 9, 10, 11] together with its physical consequences. (In Ref. [11] this FI term is derived in a simpler way by utilizing a superfield approach 15] to 5D conformal supergravity.) As for the second FI term of $Y^{Z}$, it involves the product of two $Z_{2}$-odd fields, $\beta$ and $Y^{Z(3)}$, and thus depends on how to regulate the behavior of these $Z_{2}$-odd fields across the boundary. However, while the first term is $\mathcal{O}\left(\xi_{F I}\right)$, the second term is $\mathcal{O}\left(\xi_{F I}^{2}\right)$ since $\beta=\mathcal{O}\left(\xi_{F I}\right)$ near the boundary, thus can be ignored when $\xi_{F I} \ll M_{5}$ which is the limit that orbifold SUGRA does make sense.

In case with gauged $U(1)_{R}$ symmetry, there exist additional FI terms as expected. When $r \neq 0$ and/or $k \neq 0$, the term $2 \operatorname{tr}\left[Y^{I^{\dagger}} \mathcal{A}^{\dagger} t_{I} \mathcal{A}\right]$ in our bulk Lagrangian (2.5) gives a bulk FI term for $Y^{X}$ and/or $Y^{Z}$ after the gauge fixing (2.10):

$$
2 r \operatorname{tr}\left[i \sigma_{3} Y^{X}\right]+3 k \epsilon(y) \operatorname{tr}\left[i \sigma_{3} Y^{Z}\right] .
$$

Integrating out $Y^{Z}$ then leads to a bulk cosmological constant $-3 k^{2} / 2 \alpha$ with which the total bulk cosmological constant is given by $-k^{2}\left(3 \alpha^{-1}+9 \alpha^{2}\right) / 2=-6 k^{2}+\mathcal{O}\left(k^{2} \phi^{2}\right)$. Also as a consequence of (2.4), the bulk FI term of $Y^{Z}$ appears together with the boundary tension term: $3 \alpha k \partial_{y} \epsilon(y)=\left(3 k+\mathcal{O}\left(k \phi^{2}\right)\right) \partial_{y} \epsilon(y)$. When $r \neq 0$, there can be additional boundary FI terms of $Y^{X}$. To see this, let us briefly review the construction of 4D SUGRA at the boundaries in 5D orbifold SUGRA [7]. The general boundary Lagrangian can be written as

$$
\mathcal{L}_{\mathrm{N}=1}=\sum_{l=0, \pi} \Lambda_{l} \delta\left(y-y_{l}\right)\left(-\frac{3}{2}\left[\Sigma \bar{\Sigma} e^{-K^{(l)}(S, \bar{S}) / 3}\right]_{D}+\left[f_{I J}^{(l)}(S) W^{I \alpha} W_{\alpha}^{J}\right]_{F}+\left[\Sigma^{3} W^{(l)}(S)\right]_{F}(2 ., 14)\right.
$$

where $\left(y_{0}, y_{\pi}\right)=(0, \pi R)$ and $\Lambda_{0, \pi}$ are constants. $\Sigma$ is the $4 \mathrm{D} N=1$ compensator chiral multiplet induced by the $5 \mathrm{D}$ compensator hypermultiplet $\mathcal{H}_{c}$, and $S$ and $W^{I \alpha}$ stand for generic chiral matter and gauge multiplets at the boundaries which come from either bulk fields or pure boundary fields. Here the subscripts $D$ and $F$ represent the $D$ - and $F$-components, respectively, in the $4 \mathrm{D}$ superconformal tensor calculus [7]. Let $\Sigma=\left(z^{0}, \chi_{R}^{0}, F^{0}\right)$ denote the component fields of the $N=1$ compensator superfield. The bosonic components of $\Sigma$ are given by []]

$$
\begin{aligned}
z^{0} & =\left(\mathcal{A}_{+}^{*}\right)^{2 / 3}, \\
F^{0} & =\frac{2}{3} i\left(\mathcal{A}_{+}^{*}\right)^{2 / 3} e_{4}^{y}\left(V_{y}^{N(1)}+i V_{y}^{N(2)}\right)+\frac{2}{3}\left(1+i \alpha^{-1} e_{4}^{y} A_{y}^{Z}\right)\left(\mathcal{A}_{+}^{*}\right)^{-1 / 3}\left(\tilde{\mathcal{F}}^{(1)}+i \tilde{\mathcal{F}}^{(2)}\right),
\end{aligned}
$$

where $\mathcal{A}_{+} \equiv\left(\mathcal{A}_{i=2}^{x=2}\right)^{*}=\sqrt{1+\operatorname{tr}\left[\Phi^{\dagger} \Phi\right] / 2}, V_{\mu}^{N}$ is the auxiliary $S U(2)_{\mathbf{U}}$ gauge field redefined as (2.11) , and $\tilde{\mathcal{F}} \equiv \mathcal{F}-\mathcal{F}_{\text {sol }}=\mathcal{F}-\alpha t_{Z} \mathcal{A}$. Here we use the notation $V_{\mu}^{N}=\sum_{r=1}^{3}\left(i \sigma_{r}\right) V_{\mu}^{N(r)}$ and $\tilde{\mathcal{F}}=\tilde{\mathcal{F}}^{(0)} \mathbf{1}_{2}-\sum_{r=1}^{3}\left(i \sigma_{r}\right) \tilde{\mathcal{F}}^{(r)}$. Using the standard tensor calculus, it is straightforward to find the $D$-component of the real superfield $\Sigma \bar{\Sigma}$ :

$$
\left.\Sigma \bar{\Sigma}\right|_{D} ^{\text {bosonic }}=\frac{4}{3} r\left|z^{0}\right|^{2}\left(2 Y^{X(3)}-e_{4}^{y} \partial_{y} \beta\right)+2\left(\left|F^{0}\right|^{2}+\left|\hat{\mathcal{D}}_{\underline{\mu}}^{(4)} z^{0}\right|^{2}\right),
$$

where

$$
\hat{\mathcal{D}}_{\underline{\mu}}^{(4)} z^{0}=\frac{2}{3} z_{0}^{-1 / 2}\left(\partial_{\underline{\mu}} \mathcal{A}_{+}^{*}-i\left(V_{\underline{\mu}}^{N(3)}+e_{4}^{y} v_{\underline{\mu}}\right) \mathcal{A}_{+}^{*}\right) .
$$


Here we omit all the fermionic degrees of freedom and also ignored the terms involving $Z_{2}$-odd fields which either vanish at the boundaries or are irrelevant for the following discussion.

As a minimal example of the boundary SUGRA, let us consider the pure 4D SUGRA on the boundaries which corresponds to the case with $K^{(0, \pi)}=f_{I J}^{(0, \pi)}=W^{(0, \pi)}=0$ in (2.14). Then the bosonic part of the boundary SUGRA is given by

$$
\begin{aligned}
\mathcal{L}_{N=1}= & -\frac{3}{2}[\Sigma \bar{\Sigma}]_{D}^{\text {bosonic }}\left(\Lambda_{0} \delta(y)+\Lambda_{\pi} \delta(y-\pi R)\right) \\
= & -e_{(4)} M_{(4)}^{2}\left[\frac{1}{2} R^{(4)}+2 r\left(2 Y^{X(3)}-e_{4}^{y} \partial_{y} \beta\right)\right. \\
& \left.+3\left|z^{0}\right|^{-2}\left(\left|F^{0}\right|^{2}+\left|\hat{\mathcal{D}}_{\underline{\mu}}^{(4)} z^{0}\right|^{2}\right)\right]\left(\Lambda_{0} \delta(y)+\Lambda_{\pi} \delta(y-\pi R)\right),
\end{aligned}
$$

where $R^{(4)}$ is the induced Ricci-scalar on the boundaries and

$$
M_{(4)}^{2}=\left(M_{5}^{3}+\operatorname{tr}\left[\Phi^{\dagger} \Phi\right] / 2\right)^{2 / 3}
$$

where we recover the $5 \mathrm{D}$ Planck mass $M_{5}$ which was set as $M_{5}=1$ in the previous discussion. The above boundary SUGRA contains a Fayet-Iliopoulos term

$$
-2 r M_{(4)}^{2}\left(2 Y^{X(3)}-e_{4}^{y} \partial_{y} \beta\right)\left(\Lambda_{0} \delta(y)+\Lambda_{\pi} \delta(y-\pi R)\right) .
$$

Note that unlike the boundary FI term (2.12) from the $\xi_{F I}$-term in $\tilde{N}$, these boundary FI terms from $U(1)_{R}$ gauging can have independent coefficients at different boundaries, i.e. $\Lambda_{0}$ and $\Lambda_{\pi}$ are independent from each other.

The total action of our $U(1)_{R}$-gauged SUGRA including the minimal boundary SUGRA (2.15) is given by

$$
\mathcal{L}=\mathcal{L}_{\text {bulk }}+\mathcal{L}_{\text {brane }}+\mathcal{L}_{\text {aux }}+\mathcal{L}_{N=1}
$$

In the presence of $\mathcal{L}_{N=1}$, the on-shell values of the auxiliary fields $Y^{I}, V_{\mu}^{N}, \tilde{\mathcal{F}}=\mathcal{F}-\alpha t_{Z} \mathcal{A}$, $v_{\mu \nu}$ are found to be

$$
\begin{aligned}
Y^{I} & =\tilde{\mathcal{N}}^{I J} \tilde{\mathcal{Y}}_{J}, \quad V_{\underline{\mu}}^{N(1),(2)}=V_{\underline{\mu} \mathrm{sol}}^{(1),(2)}, \quad V_{y}^{N}=V_{y \mathrm{sol}}+\Delta V_{y \mathrm{sol}}, \\
V_{\underline{\mu}}^{N(3)} & =\frac{2+\Delta_{D}}{2+3 \Delta_{D}} V_{\underline{\mu} \mathrm{sol}}^{(3)}-\frac{2 \Delta_{D}}{2+3 \Delta_{D}} e_{4}^{y} v_{\mu y \mathrm{sol}}, \quad \tilde{\mathcal{F}}^{(0),(3)}=0, \\
\tilde{\mathcal{F}}^{(1)} & =\frac{\mathcal{A}_{+}^{-1} \Delta_{D}}{1+\left(\mathcal{A}_{+}^{-2}-1\right) \Delta_{D}} \frac{e_{4}^{y}\left(V_{y \mathrm{sol}}^{(2)}-\alpha^{-1} e_{4}^{y} A_{y}^{Z} V_{y \mathrm{sol}}^{(1)}\right)}{1-\alpha^{-2} A_{\mu}^{Z} A^{Z \mu}}, \\
\tilde{\mathcal{F}}^{(2)} & =-\frac{\mathcal{A}_{+}^{-1} \Delta_{D}}{1+\left(\mathcal{A}_{+}^{-2}-1\right) \Delta_{D}} \frac{e_{4}^{y}\left(V_{y \mathrm{sol}}^{(1)}+\alpha^{-1} e_{4}^{y} A_{y}^{Z} V_{y \mathrm{sol}}^{(2)}\right)}{1-\alpha^{-2} A_{\mu}^{Z} A^{Z \mu}}, \\
v_{\underline{\mu} \underline{\underline{\mu}}} & =v_{\underline{\mu} \underline{\underline{s} \mathrm{sol}}}, \quad v_{\underline{\mu} y}=\frac{2+2 \Delta_{D}}{2+3 \Delta_{D}} v_{\underline{\mu} y \mathrm{sol}}-\frac{\Delta_{D}}{2+3 \Delta_{D}} e_{y}^{4} V_{\underline{\mu} \mathrm{sol}}^{(3)},
\end{aligned}
$$


where

$$
\begin{aligned}
\tilde{\mathcal{Y}}_{I}= & 2\left(\mathcal{A}^{\dagger}\left(t_{I}\right) \mathcal{A}-\Phi^{\dagger}\left(t_{I}\right) \Phi\right)-\frac{1}{2} \xi_{F I} e^{-1} e_{(4)}\left(i \sigma_{3}\right) \alpha^{2} \delta_{I}^{X}(\delta(y)-\delta(y-\pi R)) \\
& -2 r M_{(4)}^{2}\left(i \sigma_{3}\right) \delta_{I}^{X}\left(\Lambda_{0} \delta(y)+\Lambda_{\pi} \delta(y-\pi R)\right), \\
\Delta V_{y \text { sol }}= & \frac{\Delta_{D}}{1+\left(\mathcal{A}_{+}^{-2}-1\right) \Delta_{D}}\left(\left(i \sigma_{1}\right) V_{y \text { sol }}^{(1)}+\left(i \sigma_{2}\right) V_{y \text { sol }}^{(2)}\right), \\
\Delta_{D}= & \frac{2}{3} e^{-1} e_{(4)} M_{(4)}^{2}\left(\Lambda_{0} \delta(y)+\Lambda_{\pi} \delta(y-\pi R)\right),
\end{aligned}
$$

and $V_{\mu \mathrm{sol}}=\sum_{r=1}^{3}\left(i \sigma_{3}\right) V_{\mu \mathrm{sol}}^{(r)}$ and $v_{\mu \nu \text { sol }}$ are defined as Eq. (2.8). The part of (2.18) which corresponds to the $5 \mathrm{D}$ scalar potential is given by

$$
\begin{aligned}
V_{5 D}= & \operatorname{tr}\left[M^{I} M^{J}\left\{\Phi^{\dagger} t_{I}^{\dagger} t_{J} \Phi-\mathcal{A}^{\dagger} t_{I}^{\dagger} t_{J} \mathcal{A}\right\}-\frac{1}{2} \tilde{\mathcal{N}}^{I J} \tilde{\mathcal{Y}}_{I}^{\dagger} \tilde{\mathcal{Y}}_{J}\right] \\
& +2 e^{-1} e_{(4)} \alpha\left(3 k+\frac{3}{2} k \operatorname{tr}\left[\Phi^{\dagger} \Phi\right]+c \operatorname{tr}\left[\Phi^{\dagger} \sigma_{3} \Phi \sigma_{3}\right]\right)(\delta(y)-\delta(y-\pi R)) .
\end{aligned}
$$

\section{4D Poincaré invariant solutions}

In this section, we discuss $4 \mathrm{D}$ Poincaré invariant solutions of the $U(1)_{R}$-gauged orbifold SUGRA presented in the previous section. We first derive the Killing spinor equations and the energy functional for generic 4D Poincaré invariant metric:

$$
d s^{2}=e^{2 K(y)} \eta_{\underline{\mu} \underline{ }}(x) d x^{\underline{\mu}} d x^{\underline{\nu}}-d y^{2} .
$$

and then consider some physical implications of the $U(1)_{R}$ FI terms associated with a $U(1)_{R}$ charge $r \neq 0$.

\subsection{Killing conditions and 4D energy functional}

Applying the local SUSY transformations of the gravitinos, gauginos, and the compensator and physical hyperinos [7], we find the corresponding Killing spinor conditions:

$$
\begin{aligned}
& \kappa \equiv \partial_{y} K-\frac{1}{3} M^{I} \tilde{\mathcal{Y}}_{I}\left(i \sigma_{3}\right)^{\dagger}=0 \\
& G^{I} \equiv \partial_{y} M^{I}-2\left(\tilde{\mathcal{N}}^{I J}-\frac{1}{6} M^{I} M^{J}\right) \tilde{\mathcal{Y}}_{J}=0 \\
& F \equiv \partial_{y} \Phi-\Phi\left(V_{y}^{N}\right)^{\dagger}-M^{I}\left(g t_{I}\right) \Phi\left(i \sigma_{3}\right)^{\dagger}+\frac{1}{2} \Phi M^{I} \tilde{\mathcal{Y}}_{I}\left(i \sigma_{3}\right)^{\dagger}=0 \\
& \tilde{F} \equiv \partial_{y} \mathcal{A}-\mathcal{A}\left(V_{y}^{N}\right)^{\dagger}-\tilde{\mathcal{F}}\left(\left(i \sigma_{3}\right)^{\dagger}+\alpha^{-1} A_{y}^{Z}\right) \\
& \quad-M^{I}\left(g t_{I}\right) \mathcal{A}\left(i \sigma_{3}\right)^{\dagger}+\frac{1}{2} \mathcal{A} M^{I} \tilde{\mathcal{Y}}_{I}\left(i \sigma_{3}\right)^{\dagger}=0
\end{aligned}
$$

where

$$
\begin{aligned}
\tilde{\mathcal{Y}}_{Z}= & -2 \epsilon(y)\left(\frac{3}{2} k \mathcal{A}^{\dagger}\left(i \sigma_{3}\right) \mathcal{A}+c \Phi^{\dagger}\left(i \sigma_{3}\right) \Phi\right) \\
\tilde{\mathcal{Y}}_{X}= & -2\left(r \mathcal{A}^{\dagger}\left(i \sigma_{3}\right) \mathcal{A}+q \Phi^{\dagger}\left(i \sigma_{3}\right) \Phi\right)-\frac{1}{2} g \xi_{F I} \alpha^{2}\left(i \sigma_{3}\right)(\delta(y)-\delta(y-\pi R)) \\
& -2 r M_{(4)}^{2}\left(i \sigma_{3}\right)\left(\Lambda_{0} \delta(y)+\Lambda_{\pi} \delta(y-\pi R)\right)
\end{aligned}
$$


and $M_{(4)}^{2}$ defined in Eq. (2.16). When all the above Killing conditions are satisfied, there can be unbroken $4 \mathrm{D} N=1$ supersymmetry with the corresponding Killing spinor

$$
\epsilon_{+}(y)=\exp \left[\frac{1}{2}(K(y)-K(0)) \mathbf{1}_{2}+\int_{0}^{y} d z V_{y}^{N}(z)\right] \epsilon_{+}(0),
$$

where $\epsilon_{+}=\left(\epsilon_{R}^{i=1}, \epsilon_{L}^{i=2}\right)$ and $\epsilon^{i}$ is the 5D supersymmetry transformation parameter.

The $4 \mathrm{D}$ energy density of a $4 \mathrm{D}$ Poincaré invariant configuration is given by

$$
\begin{aligned}
E=\int d y e^{4 K} \operatorname{tr}[ & \frac{1}{4} a_{I J} G^{I \dagger} G^{J}-3|\kappa|^{2}+|F|^{2}-|\tilde{F}|^{2}+|\Delta F|^{2}-|\Delta \tilde{F}|^{2} \\
& \left.\quad-\left(F^{\dagger} \Delta F-\tilde{F}^{\dagger} \Delta \tilde{F}+\text { h.c. }\right)-2\left|V_{y \text { sol }}\right|^{2}-\frac{1}{2} M^{I} V_{y \text { sol }}\left[\mathcal{Y}_{I}, i \sigma_{3}\right]+\Delta \mathcal{E}\right],
\end{aligned}
$$

where

$$
\begin{aligned}
& \Delta F=-\Phi \Delta V_{y \text { sol }}^{\dagger}, \quad \Delta \tilde{F}=-\mathcal{A} \Delta V_{y \text { sol }}^{\dagger}-\tilde{\mathcal{F}}\left(\left(i \sigma_{3}\right)^{\dagger}+\alpha^{-1} A_{y}^{Z} \mathbf{1}_{2}\right), \\
& \Delta \mathcal{E}=\frac{\Delta_{D}}{1+\left(\mathcal{A}_{+}^{-2}-1\right) \Delta_{D}}\left(\left(V_{y \text { sol }}^{(1)}\right)^{2}+\left(V_{y \text { sol }}^{(2)}\right)^{2}\right) \mathbf{1}_{2},
\end{aligned}
$$

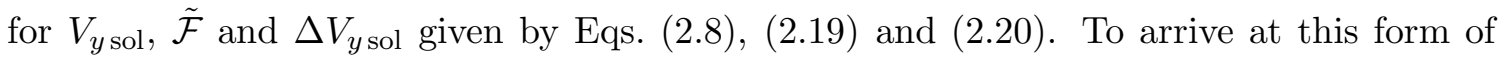
$4 \mathrm{D}$ energy density starting from the $5 \mathrm{D}$ action (2.18), we have truncated the UV sensitive higher-order boundary operators of $\mathcal{O}\left(\lambda \xi_{F I}^{2}\right), \mathcal{O}\left(\lambda \Lambda_{0, \pi}^{2}\right)\left(\lambda=\left(\xi_{F I}, \Lambda_{0, \pi}, k, c\right)\right)$ whose precise value depend on how to regulate the $Z_{2}$-odd fields at the boundaries. The above form of the $4 \mathrm{D}$ energy density indicates that a field configuration satisfying the Killing conditions:

$$
\kappa=G^{I}=F=\tilde{F}=0,
$$

as well as the stationary conditions:

$$
V_{y \mathrm{sol}}=0, \quad\left[\mathcal{Y}_{I}, \sigma_{3}\right]=0
$$

corresponds to a supersymmetric solution with vanishing vacuum energy. A simple solution of the above stationary conditions is

$$
\Phi=\left(\begin{array}{cc}
v(y) & 0 \\
0 & v(y)
\end{array}\right),
$$

where $v$ is a real function of $y$.

If $\xi_{F I}=0$, the gaugino Killing conditions are simplified as

$$
G^{I} \equiv \frac{\partial M^{I}}{\partial \phi} D=\frac{\partial M^{I}}{\partial \phi}\left[\partial_{y} \phi+g^{\phi \phi} \frac{\partial M^{I}}{\partial \phi} \tilde{\mathcal{Y}}_{I}\left(i \sigma_{3}\right)^{\dagger}\right]=0
$$

Since the physical implications of the FI coefficient $\xi_{F I}$ have been studied extensively before [8, 9, 10, 11], here we restrict ourselves to the case of $\xi_{F I}=0$ but $r \neq 0$, while leaving the more general case with $\xi_{F I} \neq 0$ and $r \neq 0$ for future work. When $\xi_{F I}=0$, 
the Killing conditions and the form of the 4D energy density can be simplified under the ansatz (3.3) for the physical hyperscalar field. In this situation, we have

$$
M^{I} \tilde{\mathcal{Y}}_{I}\left(i \sigma_{3}\right)^{\dagger}=\mathcal{P} \mathbf{1}_{2}, \quad \frac{\partial M^{I}}{\partial \phi} \tilde{Y}_{I}\left(i \sigma_{3}\right)^{\dagger}=\partial_{\phi}(\mathcal{P}+\Xi) \mathbf{1}_{2}
$$

where

$$
\begin{aligned}
& \mathcal{P} \equiv-2\left[\left(\frac{3}{2} k \epsilon(y) \alpha+r \beta\right)+v^{2}\left(\left(\frac{3}{2} k+c\right) \epsilon(y) \alpha+(r+q) \beta\right)\right] \\
& \Xi \equiv-2 r M_{(4)}^{2} \beta\left(\Lambda_{0} \delta(y)+\Lambda_{\pi} \delta(y-\pi R)\right)
\end{aligned}
$$

where we again ignore the UV-sensitive higher-dimensional boundary operators. We then find the Killing parameters:

$$
\begin{aligned}
\kappa & =\partial_{y} K-\frac{1}{3} \mathcal{P} \\
D & =\partial_{y} \phi+g^{\phi \phi} \partial_{\phi}(\mathcal{P}+\Xi) \\
F & =\partial_{y} v-v\left(q \beta+c \epsilon(y) \alpha-\frac{1}{2} \mathcal{P}\right), \\
\tilde{F} & =\frac{v}{\sqrt{1+v^{2}}} F
\end{aligned}
$$

and also the 4D energy density:

$$
E=\int d y e^{4 K}\left[\frac{1}{2} g_{\phi \phi} D^{2}+\frac{2}{1+v^{2}} F^{2}-6 \kappa^{2}\right],
$$

which tells us that the field configuration satisfying

$$
\kappa=D=F=0,
$$

corresponds to a supersymmetric vacuum solution of the theory.

\subsection{Vacuum solutions with $U(1)_{R}$ FI terms}

In this subsection, we discuss some aspects of the vacuum solution in gauged $U(1)_{R}$ SUGRA on $S^{1} / Z_{2}$. Before going to the main analysis, we briefly discuss the condition for the $U(1)_{R}$ anomaly cancellation. To be complete, let us introduce boundary chiral multiplets

$$
S_{0}^{a}=\left(z_{0}^{a}, \chi_{0}^{a}, f_{0}^{a}\right), \quad S_{\pi}^{\alpha}=\left(z_{\pi}^{\alpha}, \chi_{\pi}^{\alpha}, f_{\pi}^{\alpha}\right),
$$

confined at $y=0$ and $y=\pi R$, respectively, and let $q_{0}^{a}$ and $q_{\pi}^{\alpha}$ denote the $U(1)_{X}$ charge of their scalar components $z_{0}^{a}$ and $z_{\pi}^{\alpha}$, respectively. Here $\chi_{0}^{a}, \chi_{\pi}^{\alpha}$ and $f_{0}^{a}, f_{\pi}^{\alpha}$ denote the chiral fermion and the complex auxiliary components of $S_{0}^{a}, S_{\pi}^{\alpha}$, respectively. Then the $U(1)_{R}^{3}$ and $U(1)_{R}$-gravity-gravity anomaly cancellation conditions are given by

$$
\begin{aligned}
& (3+1) r^{3}+\sum_{\text {gaugino }} r^{3}+\sum_{\text {bulk }} q^{3}+\sum_{a}\left(q_{0}^{a}-r\right)^{3}+\sum_{\alpha}\left(q_{\pi}^{\alpha}-r\right)^{3}=0, \\
& (-21+1) r+\sum_{\text {gaugino }} r+\sum_{\text {bulk }} q+\sum_{a}\left(q_{0}^{a}-r\right)+\sum_{\alpha}\left(q_{\pi}^{\alpha}-r\right)=0,
\end{aligned}
$$


where the first terms represent the contributions from the gravitino and radino zero modes.

To see the effects of $Z_{2}$-even $U(1)_{R}$-gauging, i.e., of $r \neq 0$, let us first consider the simplest situation that $\xi_{F I}=\Lambda_{0}=\Lambda_{\pi}=k=c=0$ and there is no $U(1)_{X}$-charged boundary matter fields. In this case, a unique solution of the Killing conditions (3.4) is given by $K=\phi=0$ and a constant hyperscalar VEV

$$
v=v_{0} \equiv \pm \sqrt{-\frac{r}{r+q}} .
$$

Such hyperscalar VEV is allowed as long as $q / r<-1$, which is in fact required in order for the anomaly cancellation condition (3.5) to be satisfied.

If one introduces boundary $U(1)_{R}$ FI terms $\left(\Lambda_{0, \pi} \neq 0\right)$ into the above model, the supersymmetric vacuum solution is deformed as follows. The corresponding Killing spinor conditions are given by

$$
\begin{aligned}
\partial_{y} K & =-\frac{2 \sqrt{2}}{3}\left(r+(r+q) v^{2}\right) \cosh ^{2 / 3}(\phi) \tanh (\phi), \\
\partial_{y} \phi & =2 I(\phi)\left[r+(r+q) v^{2}+r M_{(4)}^{2}(v)\left(\Lambda_{0} \delta(y)+\Lambda_{\pi} \delta(y-\pi R)\right)\right], \\
\partial_{y} v & =\sqrt{2}(r+q)\left(1+v^{2}\right) v \cosh ^{2 / 3}(\phi) \tanh (\phi),
\end{aligned}
$$

where

$$
I(\phi)=\frac{\sqrt{2} \cosh ^{2 / 3}(\phi)(2+\cosh (2 \phi))}{1+2 \cosh (2 \phi)} .
$$

Obviously, the existence of $\Lambda_{0} \delta(y)+\Lambda_{\pi} \delta(y-\pi R)$ in the gaugino Killing condition enforces $\phi(y)$ to have a non-trivial $y$-dependence, and thus $v(y)$ also. In the limit that $|\phi(y)| \ll 1$ over the entire orbifold, which would be the case if $|r|,|q| \ll 1$ (in the unit with $M_{5}=1$ ), the gaugino and hyperino Killing conditions can be approximated as

$$
\begin{aligned}
& \partial_{y} \phi \simeq 2 \sqrt{2}\left[r+(r+q) v^{2}+r M_{(4)}^{2}(v)\left(\Lambda_{0} \delta(y)+\Lambda_{\pi} \delta(y-\pi R)\right)\right], \\
& \partial_{y} v \simeq \sqrt{2}(r+q) v\left(1+v^{2}\right) \phi .
\end{aligned}
$$

Then at leading order in $\delta v \equiv v-v_{0}\left(v_{0}= \pm \sqrt{-r /(r+q)}\right)$ which is presumed to be a small vacuum deformation, we find

$$
\begin{aligned}
v & =v_{0}+\left(A e^{\omega y}+B e^{-\omega y}\right), \\
\phi & =-2 \frac{\left|r v_{0}\right|}{r v_{0}} \sqrt{1+\frac{r}{q}}\left(A e^{\omega y}-B e^{-\omega y}\right),
\end{aligned}
$$

for $0<y<\pi R$, where $\omega=\sqrt{-8 r q}$ and

$$
\begin{aligned}
& A=\frac{\sqrt{2}\left|r v_{0}\right|}{2 v_{0}}\left(\frac{q}{r+q}\right)^{7 / 6}\left(\frac{\Lambda_{0}+\Lambda_{\pi} e^{\omega \pi R}}{e^{2 \omega \pi R}-1}\right), \\
& B=\frac{\sqrt{2}\left|r v_{0}\right|}{2 v_{0}}\left(\frac{q}{r+q}\right)^{7 / 6}\left(\frac{\Lambda_{0}+\Lambda_{\pi} e^{-\omega \pi R}}{1-e^{-2 \omega \pi R}}\right) .
\end{aligned}
$$




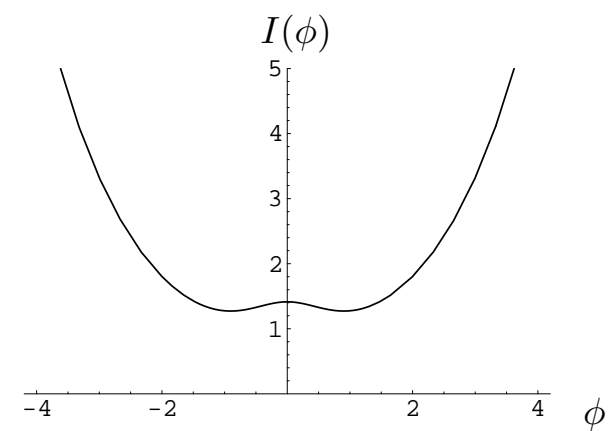

Figure 1: Numerical value of $I(\phi)$ for $|\phi| \leq 4$.

The above relations between the integration constants $A, B$ and the orbifold radius $R$ show that once there exists a dynamics to determine one (or both) of the boundary values of $v(y)$, e.g., the boundary superpotentials of $\Phi$ which would determine $v(0)=v_{0}+A+B$ and/or $v(\pi R)=v_{0}+A e^{\omega \pi R}+B e^{-\omega \pi R}$, one might be able to fix $R$ as well as to break $N=1$ SUSY through the combined effects of the $U(1)_{R}$ FI terms and the boundary superpotentials. Note that both $\phi(y)$ and $\delta v(y)$ are small over the entire orbifold when $|r|,|q| \ll 1$, justifying the use of the approximate Killing conditions (3.7).

As another example, we can consider the case that the $D$-flat condition, i.e., the gaugino Killing condition, is satisfied through the nonzero VEVs of the boundary chiral multiplets $S_{0}^{a}$ and/or $S_{\pi}^{\alpha}$, while the bulk hyperscalars have vanishing VEVs. For simplicity, let us again set $\xi_{F I}=k=c=0$, while keeping $r$ and $\Lambda_{0, \pi}$ to be nonzero, and assume the minimal form of the boundary Kähler potentials: $K^{(0)}\left(S_{0}, \bar{S}_{0}\right)=\sum_{a} \bar{S}_{0}^{a} S_{0}^{a}$ and $K^{(\pi)}\left(S_{\pi}, \bar{S}_{\pi}\right)=\sum_{\alpha} \bar{S}_{\pi}^{\alpha} S_{\pi}^{\alpha}$. Then the gravitino and gaugino Killing conditions are given by

$$
\begin{aligned}
\partial_{y} K & =-\frac{2 \sqrt{2}}{3} r \cosh ^{2 / 3}(\phi) \tanh (\phi), \\
\partial_{y} \phi & =2 I(\phi)\left[r+\lambda_{0} \delta(y)+\lambda_{\pi} \delta(y-\pi R)\right],
\end{aligned}
$$

where $\lambda_{0}=\left(r+\sum_{a} q_{0}^{a}\left|z_{0}^{a}\right|^{2}\right) \Lambda_{0}$ and $\lambda_{\pi}=\left(r+\sum_{\alpha} q_{\pi}^{\alpha}\left|z_{\pi}^{\alpha}\right|^{2}\right) \Lambda_{\pi}$. In the limit that $|\phi(y)| \ll 1$ over the entire orbifold, this $D$-flat condition leads to

$$
\begin{aligned}
\phi & \simeq 2 \sqrt{2} r y+\sqrt{2} \lambda_{0} \\
e^{2 K} & \simeq \exp \left(-\frac{8}{3} r^{2} y^{2}-\frac{8}{3} \lambda_{0} r y\right),
\end{aligned}
$$

for $0<y<\pi R$. Although derived under the condition that $|\phi(y)| \ll 1$ for $0<y<\pi R$, the above approximate solutions are valid as long as $|\phi(y)| \lesssim 1$ for which $I(\phi)$ is approximately a constant as can be seen from Fig. 1. (If $|\phi| \gtrsim 1$ near the boundary, the resulting boundary fluctuations of $\beta(\phi)$ would be too large to be described by orbifold field theory.) In this case, the integrability condition $\oint d y \partial_{y} \phi=0$ determines the orbifold radius (for given values of $\left.\lambda_{0, \pi}\right)$ as

$$
2 \pi R \simeq-\frac{\lambda_{0}+\lambda_{\pi}}{r}
$$



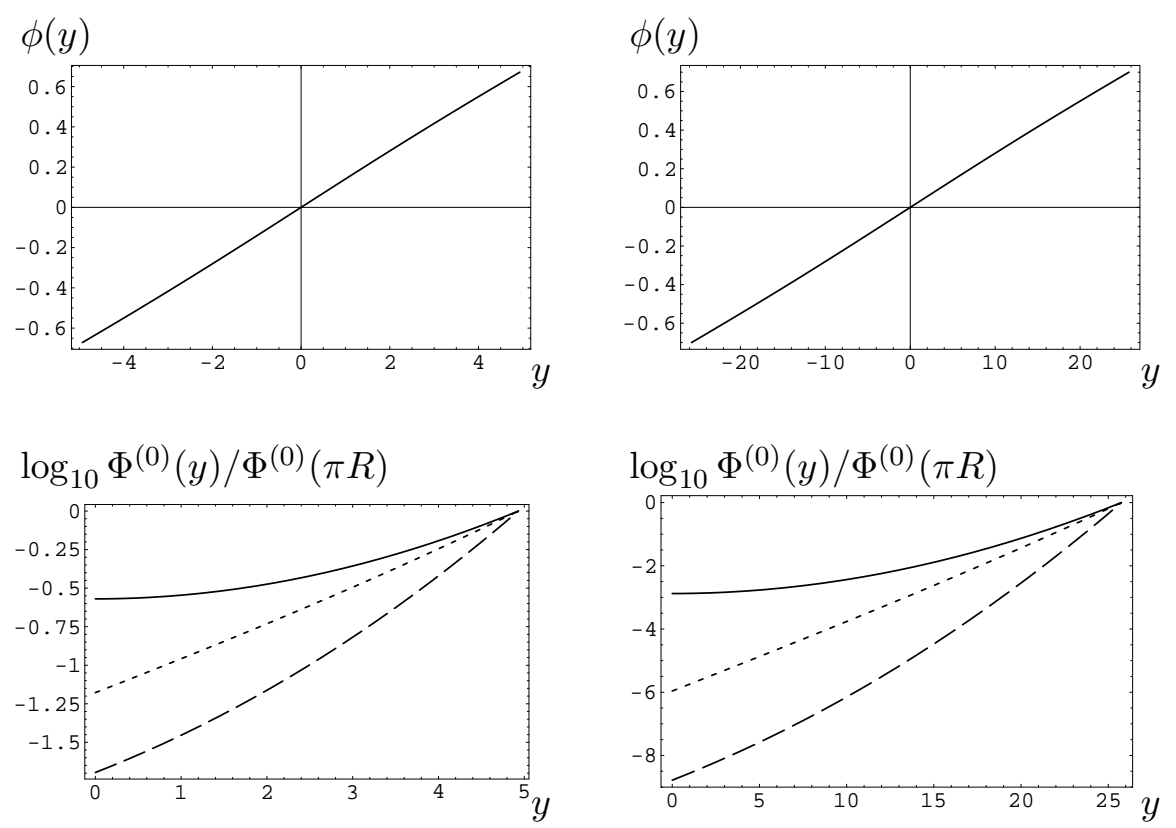

(a) $r=0.05, k=0$

(b) $r=0.01, k=0$

Figure 2: The profiles of $\phi$ and the matter zero mode $\Phi^{(0)}$ for some cases with $k=0$ and $\lambda_{0}=0$. Here we choose $\lambda_{\pi}=(r-1) / 2$. For the matter zero mode profile, the solid-, dotted- and dashedcurves represent the case with $(q, c)=(0.5,0),(0,0.5)$ and $(0.5,0.5)$, respectively. All parameters are given in the unit with $M_{5}=1$, and all the curves are shown within $|y| \leq \pi R$.

Since $\left|\lambda_{0}+\lambda_{\pi}\right| \lesssim 1$ in order for the orbifold field theory description to be valid, the above relation indicates that $2 \pi R \lesssim 1 /|r|$ for which the warp factor $e^{2 K}$ is approximately a constant. It also implies that once there exists a dynamics to determine the VEVs of the boundary scalar fields $z_{0}^{a}$ and $z_{\pi}^{\alpha}$, e.g., the boundary superpotentials, one might be able to fix $R$ through the combined effects of the $U(1)_{R}$ FI terms and the boundary superpotentials.

However the nontrivial profile of $\phi$ due to the $Z_{2}$-even $U(1)_{R}$ gauging can significantly alter the shape of the zero-mode wavefunction $\Phi^{(0)}(y)$ of a $U(1)_{X}$-charged hypermultiplet. To see this, let us consider a matter hypermultiplet with nonzero hyperino $U(1)_{X}$ charge $q(q r>0)$ and vanishing VEV. The corresponding zero mode obeys

$$
\partial_{y} \Phi^{(0)}(y)-m(y) \Phi^{(0)}(y)=0
$$

where

$$
m(y)=(3 k / 2+c) \epsilon(y) \alpha(\langle\phi(y)\rangle)+(q+r) \beta(\langle\phi(y)\rangle),
$$

in most general situation. For $\xi_{F I}=k=c=0$ which leads to $\phi(y)$ given by (3.10), one easily finds

$$
\Phi^{(0)}(y) \simeq \Phi^{(0)}(0) \exp \left[2(q+r)\left(r y^{2}+\lambda_{0} y\right)\right]
$$



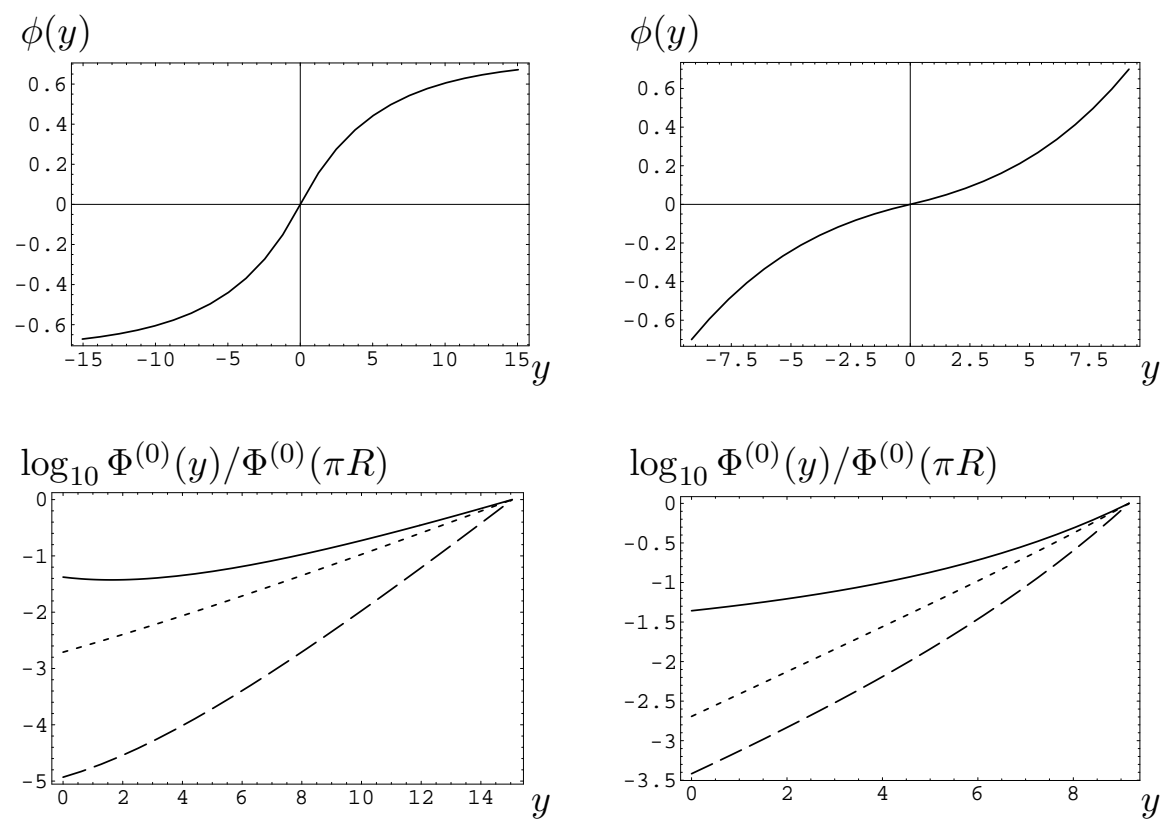

(a) $r=0.05, k=-0.1$

(b) $r=0.01, k=0.1$

Figure 3: The profiles of $\phi$ and $\Phi^{(0)}$ for $r, k \neq 0, \lambda_{0}=0$ and $\lambda_{\pi}=(r-1) / 2$. Again the solid-, dotted- and dashed-curves represent the case $(q, c)=(0.5,0),(0,0.5)$ and $(0.5,0.5)$, respectively. Note that $K \simeq-k y$ in this supersymmetric solution.

For $\pi R\left|q\left(\lambda_{0}-\lambda_{\pi}\right)\right| \gtrsim 1$, this matter zero mode would be quasi-localized at one of the orbifold boundaries as

$$
\frac{\Phi^{(0)}(\pi R)}{\Phi^{(0)}(0)} \simeq \exp \left[(q+r) \pi R\left(\lambda_{0}-\lambda_{\pi}\right)\right]
$$

If $|q \pi R|$ is large enough, the resulting quasi-localization of matter zero modes can generate small Yukawa couplings in a natural manner as proposed in Ref. [12]. In Fig. 2, we show the profiles of $\phi(y)$ and the corresponding hypermultiplet zero mode $\Phi^{(0)}$ in some cases with $k=\lambda_{0}=0$ and $\lambda_{\pi} \neq 0$. In this analysis, we consider also the case that the bare hypermultiplet kink mass $c \epsilon(y)$ is non-vanishing. Note that $\phi(y)$ takes a linear profile yielding the Gaussian profile of the matter zero mode wavefunction. The wave-function suppression at $y=0$ caused by the gauged $U(1)_{R}$ FI terms can be as important as the effect of the bare kink mass $c \epsilon(y)$. As can be noticed from Fig. 2, one can have a stronger suppression for smaller $r$ since then a larger orbifold radius is allowed. We remark that if both $\lambda_{0}$ and $\lambda_{\pi}$ are non-vanishing, the location of the minimum of the wave-function can be shifted. The extreme case would be $\lambda_{0}=\lambda_{\pi}$ for which the minimum is located at $y=\pi R / 2$.

Fig. 3 reveals the profiles of $\phi$ and $\Phi^{(0)}$ for some cases with nonvanishing $k$. Here $\phi$ has different profile for different sign of $k$, Fig. 3(a) for $r k<0$ and (b) for $r k>0$. Due to the effect of nonvanishing $k$, the orbifold radius in Fig. 3(a) is larger than the one in Fig. 2(a), 
while the radius in Fig. 3(b) is smaller than the one in Fig. 2(b). Consequently the wavefunction suppression becomes more (less) significant for $r k<0(r k>0)$ compared to the case with $k=0$.

\section{Conclusion}

In this paper, we have studied a $5 \mathrm{D}$ gauged $U(1)_{R}$ supergravity on $S^{1} / Z_{2}$ in which both a $Z_{2}$-even $U(1)$ gauge field and the $Z_{2}$-odd graviphoton take part in the $U(1)_{R}$ gauging. Based on the off-shell 5D supergravity of Refs. [7], we examined the structure of Fayet-Iliopoulos (FI) terms allowed by such theory. As expected, introducing a $Z_{2}$-even $U(1)_{R}$ gauging accompanies new bulk and boundary FI terms in addition to the known integrable boundary FI term which could be present in the absence of any gauged $U(1)_{R}$ symmetry. The new (non-integrable) boundary FI terms originate from the $N=1$ boundary supergravity, and thus are free from the bulk supergravity structure in contrast to the integrable boundary FI term which is determined by the bulk structure of 5D supergravity [8, 9, 10, 11].

We have examined some physical consequences of the $Z_{2}$-even $U(1)_{R}$ gauging in several simple cases. It is noted that the FI terms of gauged $Z_{2}$-even $U(1)_{R}$ can lead to an interesting deformation of vacuum structure which can affect the quasi-localization of the matter zero modes in extra dimension and also the SUSY breaking and radion stabilization. Thus the 5 D gauged $U(1)_{R}$ supergravity on orbifold has a rich theoretical structure which may be useful for understanding some problems in particle physics such as the Yukawa hierarchy [12] and/or the supersymmetry breaking. When one tries to construct a realistic particle physics model within gauged $U(1)_{R}$ supergravity, the most severe constraint comes from the anomaly cancellation condition [3, 16]. In some cases the Green-Schwarz mechanism might be necessary to cancel the anomaly, which may introduce another type of FI term into the theory [17]. These issues will be studied in future works.

\section{Acknowledgement}

We would like to thank Ian-Woo Kim and Keisuke Ohashi for useful discussions and comments. This work is supported by KRF PBRG 2002-070-C00022 (HA,KC) and the Center for High Energy Physics of Kyungbook National University (KC).

\section{References}

[1] L. O’Raifeartaigh, Nucl. Phys. B 96, 331 (1975).

[2] P. Fayet and J. Iliopoulos, Phys. Lett. B 51, 461 (1974).

[3] R. Barbieri, S. Ferrara, D. V. Nanopoulos and K. S. Stelle, Phys. Lett. B 113, 219 (1982);

A. H. Chamseddine and H. K. Dreiner, Nucl. Phys. B 458, 65 (1996) [hep-ph/9504337];

D. J. Castano, D. Z. Freedman and C. Manuel, Nucl. Phys. B 461, 50 (1996)

[hep-ph/9507397]; P. Binetruy, G. Dvali, R. Kallosh and A. Van Proeyen, Class. Quant.

Grav. 21, 3137 (2004) [hep-th/0402046].

[4] M. B. Green and J. H. Schwarz, Phys. Lett. B 149, 117 (1984). 
[5] R. Altendorfer, J. Bagger and D. Nemeschansky, Phys. Rev. D 63, 125025 (2001) [hep-th/0003117]; T. Gherghetta and A. Pomarol, Nucl. Phys. B 586, 141 (2000) [hep-ph/0003129]; A. Falkowski, Z. Lalak and S. Pokorski, Phys. Lett. B 491, 172 (2000) [hep-th/0004093]; K. Choi, H. D. Kim and I. W. Kim, JHEP 0211, 033 (2002) [hep-ph/0202257]; K. Choi, H. D. Kim and I. W. Kim, JHEP 0303, 034 (2003) [hep-ph/0207013].

[6] L. Randall and R. Sundrum, Phys. Rev. Lett. 83, 3370 (1999) [hep-ph/9905221].

[7] T. Fujita, T. Kugo and K. Ohashi, Prog. Theor. Phys. 106, 671 (2001) [hep-th/0106051]; T. Kugo and K. Ohashi, Prog. Theor. Phys. 108, 203 (2002) [hep-th/0203276].

[8] D. M. Ghilencea, S. Groot Nibbelink and H. P. Nilles, Nucl. Phys. B 619, 385 (2001) [hep-th/0108184].

[9] R. Barbieri, R. Contino, P. Creminelli, R. Rattazzi and C. A. Scrucca, Phys. Rev. D 66, 024025 (2002) [hep-th/0203039].

[10] S. Groot Nibbelink, H. P. Nilles and M. Olechowski, Phys. Lett. B 536, 270 (2002) [hep-th/0203055]; S. Groot Nibbelink, H. P. Nilles and M. Olechowski, Nucl. Phys. B 640, 171 (2002) [hep-th/0205012]; D. Marti and A. Pomarol, Phys. Rev. D 66, 125005 (2002) [hep-ph/0205034]; H. Abe, T. Higaki and T. Kobayashi, Prog. Theor. Phys. 109, 809 (2003) [hep-th/0210025]; T. Hirayama and K. Yoshioka, JHEP 0401, 032 (2004) [hep-th/0311233]; T. Kobayashi and K. Yoshioka, JHEP 0411, 024 (2004) [hep-ph/0409355].

[11] F. P. Correia, M. G. Schmidt and Z. Tavartkiladze, hep-th/0410281.

[12] N. Arkani-Hamed and M. Schmaltz, Phys. Rev. D 61, 033005 (2000) [hep-ph/9903417]; E. A. Mirabelli and M. Schmaltz, Phys. Rev. D 61, 113011 (2000) [hep-ph/9912265]; D. E. Kaplan and T. M. P. Tait, JHEP 0111, 051 (2001) [hep-ph/0110126]; M. Kakizaki and M. Yamaguchi, Int. J. Mod. Phys. A 19, 1715 (2004) [hep-ph/0110266]; N. Haba and N. Maru, Phys. Rev. D 66, 055005 (2002) [hep-ph/0204069]; A. Hebecker and J. March-Russell, Phys. Lett. B 541, 338 (2002) [hep-ph/0205143]; K. Choi, D. Y. Kim, I. W. Kim and T. Kobayashi, Eur. Phys. J. C 35, 267 (2004) [hep-ph/0305024]; K. Choi, I. W. Kim and W. Y. Song, Nucl. Phys. B 687, 101 (2004) [hep-ph/0307365]; H. Abe, K. Choi, K. S. Jeong and K. i. Okumura, JHEP 0409, 015 (2004) [hep-ph/0407005].

[13] E. Bergshoeff, R. Kallosh and A. Van Proeyen, JHEP 0010, 033 (2000) [hep-th/0007044].

[14] T. Kugo and K. Ohashi, Prog. Theor. Phys. 105, 323 (2001) [hep-ph/0010288].

[15] F. Paccetti Correia, M. G. Schmidt and Z. Tavartkiladze, hep-th/0408138; H. Abe and Y. Sakamura, JHEP 0410, 013 (2004) [hep-th/0408224].

[16] N. Kitazawa, N. Maru and N. Okada, Phys. Rev. D 62, 077701 (2000) [hep-ph/9911251]; N. Kitazawa, N. Maru and N. Okada, Nucl. Phys. B 586, 261 (2000) [hep-ph/0003240].

[17] E. Dudas, T. Gherghetta and S. Groot Nibbelink, Phys. Rev. D 70, 086012 (2004) [hep-th/0404094]. 